Draft Version July 10, 2018

Preprint typeset using $\mathrm{LAT}_{\mathrm{E} X} \mathrm{X}$ style emulateapj v. 6/22/04

\title{
ANALYTICAL MODELS FOR THE ENERGETICS OF COSMIC ACCRETION SHOCKS, THEIR COSMOLOGICAL EVOLUTION, AND THE EFFECT OF ENVIRONMENT
}

\author{
VASILIKI PAVLIDOU ${ }^{1,2}$ AND BRIAN D. Fields ${ }^{2}$ \\ Draft version July 10, 2018
}

\begin{abstract}
We present an analytical description of the energetics of the population of cosmic accretion shocks, for a concordance cosmology $\left(\Omega_{\mathrm{m}}+\Omega_{\Lambda}=1\right)$. We calculate how the shock-processed accretion power and mass current are distributed among different shock Mach numbers, and how they evolve with cosmic time. We calculate the cumulative energy input of cosmic accretion shocks of any Mach number to the intergalactic medium as a function of redshift, and we compare it with the energy output of supernova explosions as well as with the energy input required to reionize the universe. In addition, we investigate and quantify the effect of environmental factors, such as local clustering properties and filament preheating on the statistical properties of these shocks. We find that the energy processed by accretion shocks is higher than the supernova energy output for all $z<3$ and that it becomes more than an order of magnitude higher in the local universe. The energy processed by accretion shocks alone becomes comparable to the energy required to reionize the universe by $z \sim 3.5$. Finally, we establish both qualitative and quantitatively that both local clustering as well as filament compression and preheating are important factors in determining the statistical properties of the cosmic accretion shock population.
\end{abstract}

Subject headings: shock waves - large-scale structure of universe - galaxies: clusters:general - intergalactic medium - methods: analytical

\section{INTRODUCTION}

The formation of shocks in the baryonic component of matter in the universe is an inevitable and integral part of the process of cosmological structure formation. The processing of gas through cosmic shocks plays a major role in the distribution of cosmic baryons and their energetics and thermodynamic properties. In particular, the initial shock heating of primordial gas leaves it optically invisible and thus contributes to the inventory of dark baryons (Hegyi \& Olive 1986; Fukugita et al. 1998; Spergel et al. 2003; Cyburt et al. 2003). A full observational accounting of dark baryons in all of their states is now emerging, with the first evidence for a large component of diffuse, low-density intergalactic gas distributed in the filaments and sheets that comprise the "cosmic web," and detected in the X-ray forest via absorption lines from highly-ionized metals (Hellsten et al. 1998; Perna \& Loeb 1998; Fang et al. 2002; Nicastro et al. 2002; Finoguenov et al. 2003; Mathur et al. 2003; Nicastro et al. 2005). This warm-hot intergalactic medium is thought to arise in structure formation shocks (e.g. Hellsten et al. 1998; Cen \& Ostriker 1999; Davé et al. 2001; Nath \& Silk 2001; Furlanetto \& Loeb 2004; Kang et al. 2005).

Moreover, structure formation shocks heat the intergalactic medium and are likely to act as acceleration sites for nonthermal particles, the "structure formation cosmic rays" (e.g. Miniati et al. 2001a,b; Brunetti et al. 2001; Fujita \& Sarazin 2001; Berrington \& Dermer 2003; Gabici \& Blasi 2003a; Brunetti et al. 2004; Kang \& Jones 2005 and references therein). Recent detec-

1 Kavli Institute for Cosmological Physics, The University of Chicago, Chicago, IL 60637

2 Center for Theoretical Astrophysics, Department of Astronomy, University of Illinois, Urbana, IL 61801 tions of nonthermal emission from galaxy clusters (e.g. Fusco-Femiano et al. 1998; Govoni et al. 2001; Bagchi et al. 2002; Feretti et al. 2004 and references therein) is consistent with inverse Compton emission by relativistic intracluster electrons. Such a cosmic ray population presumably also includes hadrons, and so would also have distinct $\gamma$-ray and light-element signatures, which are currently subjects of intense investigation (Loeb \& Waxman 2000; Totani \& Kitayama 2000; Miniati 2002; Scharf \& Mukherjee 2002; Suzuki \& Inoue 2002; Totani \& Inoue 2002; Berrington \& Dermer 2003; Gabici \& Blasi 2003b, Keshet et al. 2003; Miniati 2003; Reimer et al. 2003; Gabici \& Blasi 2004; Prodanović \& Fields 2004; Prodanović \& Fields 2005; Suzuki \& Inoue 2004; Kuo et al. 2005).

Cosmic shocks occur during different facets of structure formation and in a variety of environments, hence there are at least two distinct ways to categorize them: according to the physical processes driving them, and according to the state of the medium in which they form.

There are three principal processes associated with cosmic structure formation which result to the formation of large-scale shocks.

1. Accretion of intergalactic gas by a collapsed, virialized structure. In this case, an accretion shock is formed at the interface between virialized and diffuse gas (e.g. Bertschinger 1985a; Miniati et al. 2000) The shock is driven by the gravitational attraction exerted on the diffuse gas by the accretor and heats the accreted gas to the virial temperature.

2. Merger of two collapsed structures. In this case, a merger shock is formed at the interface between the gas components of the merging objects (e.g. 
Miniati et al. 2000; Gabici \& Blasi 2003). The shock is driven by the mutual gravitational attraction between the objects.

3. Accumulation of intergalactic gas onto large-scale cosmic filaments and sheets. Contrary to virialized structures, large-scale filaments and sheets, although overdense, are still expanding. At the edges between filaments and their surroundings, shocks form, driven by the difference in expansion velocity between the overdense filament and the underdense neighboring voids (e.g. Bertschinger 2005b). More complicated shock surfaces inside the filaments are additionally produced due to the gravity of the collapsed objects residing in the filament interior.

Shocks can also be divided according to the state of the gas passing through them, into external and internal shocks (e.g. Miniati et al. 2000; Ryu et al. 2003). External shocks process pristine material, which has never been shocked before by any of the processes described above. External shocks are mostly filament shocks, since the process of formation of individual virialized structures (associated with the other two types of shocks) occurs principally within filaments, and therefore in most cases involves gas which has already been processed at least by filament shocks. Internal shocks process gas which has already been shock-heated in the past. All merger shocks, as well as many accretion shocks, are internal shocks.

Since external shocks process colder material of lower sound speed, their Mach numbers are generally higher than those of internal shocks. However, because the gas passing through internal shocks has already been compressed, internal shocks process more mass and kinetic energy than external shocks.

In this work, we present an analytical study of the energetics and cosmic evolution of the population of of cosmic accretion shocks, in a concordance $\Omega_{\mathrm{m}}+\Omega_{\Lambda}=1$ universe. In addition, we assess the effect of environmental factors on the properties of these shocks. The environmental factors we consider are: (a) variations in the local matter density and temperature of the region in which each accretor is embedded imprinted in the primordial density field and (b) filament preheating and compression.

In order to distinguish between the effects of each environmental factor, we start from a base model which accounts for no environmental effects and regards all object as being embedded in an environment well represented by the background universe. In this case, shock properties depend solely on the mass of the accretor. To this model we then add each of the environmental factors mentioned above, each version of our model being refined by one effect with respect to the previous version. The final (full) model we construct in this way accounts for the effects of the mass distribution of accretors as well as both environmental factors considered here.

Although each effect is treated under a number of simplifying assumptions which necessarily introduce some error in our calculations, we make sure to on one hand quantify this error whenever possible and on the other hand to consistently err on the side of underestimating the effect of environment. In this sense, our results are an absolute lower limit of the effect of environmental factors on the properties of the population of cosmic accretion shocks. In addition, the trends and qualitative changes introduced to the properties of the accretion shock population are clearly identified. Any future quantitative correction to our model will only enhance such trends.

The shock properties as a function of object mass are derived from the temperature jump across the shock surface (as is the case in cosmological numerical simulations), while the underlying mass-function of the accretors is taken to be the Press-Schechter mass function. We calculate statistical quantities characteristic of the population of cosmic accretion shocks, such as the distribution of number density of objects, processed kinetic energy and shocked mass with respect to Mach number, as well as their evolution with cosmic time, and we use our findings to discuss the relative importance of cosmic accretion shocks in the energetics of the intergalactic medium.

Our paper is organized as follows. In 92 we present the formalism describing the properties of a single accretion shock around a cosmic structure. In $\$ 3$ we combine our single-shock model with different cosmological distribution functions to derive the statistical properties of the population of cosmic accretion shocks. In 4 we present the results of our model for a concordance $\Omega_{\mathrm{m}}+\Omega_{\Lambda}=1$ universe. In $\$ 5$ we discuss the differences and similarities of our approach from other analytic cosmic shock models based on the Press-Schechter approach. Finally, we summarize and discuss our findings in 6 .

\section{PROPERTIES OF A SINGLE SHOCK}

In this work, the properties of the accretion shock around a single virialized host object are derived from the temperature jump across the shock, in agreement with the method used to derive shock properties in cosmological simulations. The pre-shock temperature is taken to be an appropriately defined environmental temperature, while the post-shock temperature is taken to be the virial temperature of the host structure. However, the virial temperature is a mean quantity of the host object and does not necessarily characterize the temperature right behind the shock. To assess the error introduced in our calculation of the Mach number due to this assumption, we compared our temperature-jump technique with the Bertschinger (1985) similarity solution which does take into account the radial dependences of the postshock gas. To ensure an appropriate comparison, we applied our formalism to an Einstein-de Sitter universe and in the high-Mach limit, where the Bertschinger solution is applicable. We found that the deviation from the Bertschinger solution is at the level of $1 \%$.

Throughout this paper, we assume an adiabatic equation of state, and we consider all shocks to be nonradiative. We also assume that any individual collapsed object as well as its accretion shock are spherically symmetric. We take the accretion shock position around each structure to coincide with the virial radius of each structure.

The Mach number of a shock, $\mathcal{M}$, is defined as the ratio of the velocity of the accreted material in the shock frame to the adiabatic sound speed of the accreted material. The Mach number is related to the temperature jump across the shock through

$$
\frac{T_{2}}{T_{1}}=\frac{\left(5 \mathcal{M}^{2}-1\right)\left(\mathcal{M}^{2}+3\right)}{16 \mathcal{M}^{2}} \text {. }
$$


where $T_{1}$ and $T_{2}$ are the pre-shock and post-shock temperatures correspondingly, and we have assumed a ratio of specific heats $\gamma=5 / 3$, constant across the shock. In the limit $\mathcal{M} \gg 1$ this equation is further simplified, $\mathcal{M}=$ $\sqrt{\left(16 T_{2}\right) /\left(5 T_{1}\right)}$. The pre-shock temperature can be written in terms of the adiabatic sound speed of the pre-shock material $c_{\mathrm{s} 1}$ as $k T_{1}=\mu m_{\mathrm{p}} c_{\mathrm{s} 1}^{2} / \gamma$, where $k$ is the Boltzmann constant, $\mu$ is the mean molecular weight of the accreted gas, and $m_{\mathrm{p}}$ is the proton mass. If we also take $T_{2}$ to be the virial temperature of the accretor which has a mass $m, T_{\text {vir }}=\mu m_{\mathrm{p}} G m^{2 / 3}\left(4 \pi f_{\mathrm{c}} \rho_{\mathrm{m}, 0}\right)^{1 / 3}(1+z) /\left(5 k 3^{1 / 3}\right)$, then the ratio $T_{2} / T_{1}$ becomes

$\frac{T_{2}(m, z)}{T_{1}}=2.7 \times 10^{3} \Omega_{\mathrm{m}}\left(\frac{f_{c}}{18 \pi^{2}} \frac{m^{2}}{m_{8}^{2}}\right)^{1 / 3}(1+z)\left(\frac{15 \mathrm{~km} \mathrm{~s}^{-1}}{c_{\mathrm{s} 1}}\right)^{2}$,

where $f_{c}$ is the compression factor for a virialized object (which may vary with virialization redshift, depending on the cosmological model), $z$ is the virialization redshift, $h$ is the dimensionless Hubble parameter, and $m_{8}=5.96 \times 10^{14} h^{-1} \Omega_{\mathrm{m}} \mathrm{M}_{\odot}$ is the mass included in a sphere of comoving radius $r_{8}=8 h^{-1} \mathrm{Mpc}$ assuming the mean matter density inside the sphere to be equal to the cosmic mean.

The (baryonic) mass current, defined as the rate at which mass crosses the surface of a single accretion shock around a structure of mass $m$ at an epoch $z$, is

$$
J_{1}=\frac{\Omega_{\mathrm{b}}}{\Omega_{\mathrm{m}}} \frac{d m}{d t}=4 \pi r_{\mathrm{v}}^{2}(m) \Omega_{\mathrm{b}} \rho_{\mathrm{c}, 0}(1+z)^{3}\left(1+\delta_{\mathrm{s}}\right) \mathcal{M} c_{\mathrm{s} 1}
$$

where $r_{\mathrm{v}}$ is the virial radius of the structure,

$$
r_{\mathrm{v}}=1.4 h^{-1} \mathrm{Mpc}\left(\frac{m}{m_{8}}\right)^{1 / 3}\left(\frac{f_{\mathrm{c}}}{18 \pi^{2}}\right)^{-1 / 3}(1+z)^{-1},
$$

$\rho_{\mathrm{c}, 0}$ is the critical density at the present cosmic epoch, $\Omega_{\mathrm{b}} \rho_{\mathrm{c}, 0}(1+z)^{3}$ is the cosmic baryon density at the epoch of interest, and $\left(1+\delta_{\mathrm{s}}\right)$ is the density enhancement (with respect to the cosmic mean) just outside the shock.

Finally, the kinetic power crossing the accretion shock around a single structure of mass $m$ is $P_{1}=d E / d t=$ $0.5(d m / d t) v_{1}^{2}=0.5 J_{1} v_{1}^{2}$, or

$$
P_{1}=2 \pi r_{\mathrm{v}}(m)^{2} \Omega_{\mathrm{b}} \rho_{\mathrm{c}, 0}(1+z)^{3}\left(1+\delta_{\mathrm{s}}\right) \mathcal{M}^{3} c_{\mathrm{s} 1}^{3} .
$$

\section{PROPERTIES OF THE POPULATION OF COSMIC ACCRETION SHOCKS}

We will examine three distinct models for the cosmic accretion shock population. We will start from a "base" no-environmental-effects model, in which the properties of each accretion shock are simply a function of the accretor mass. We will then gradually build up more complex models by adding one-by-one the different environmental factors we wish to examine (local density and temperature variations originating in the primordial density field, and filament compression and preheating). In this way, we will assess the effect of each factor on the properties of the cosmic shock population. Our third, most complex model, will include the effect of both environmental factors we are addressing here.

\subsection{Model 1 (base model): no environmental effects}

If we assume that all collapsed objects accrete baryons of uniform density and temperature (the density and temperature corresponding to the mean, backgrounduniverse values of diffuse baryons), then at a given redshift, all objects of a certain mass will have accretion shocks with identical Mach numbers, and will process the same amount of mass and kinetic power per unit time. In the relations of $₫ 2, \delta_{\mathrm{s}}=0$ and $c_{\mathrm{s} 1}=15 \mathrm{~km} / \mathrm{s}$ as we will be treating only post-reionization redshifts. The mass distribution of collapsed objects will be the one described by the Press-Schechter mass function (Press \& Schechter 1974; Lacey \& Cole 1993)

$$
\frac{d n}{d m}(m, z)=\sqrt{\frac{2}{\pi}} \frac{\rho_{m, 0}}{m^{2}}\left|\frac{d \ln \sigma}{d \ln m}\right| \exp \left\{-\frac{\left[\tilde{\delta}_{c}(z)\right]^{2}}{2[\sigma(m)]^{2}}\right\} .
$$

where $\tilde{\delta}_{c}(z)$ is the linearly extrapolated overdensity of an object which collapses at redshift $z, \sigma(m)=\sqrt{S(m)}$ is the square root of the variance of the linearly extrapolated field smoothed at a mass scale $m$, and $\rho_{m, 0}$ is the cosmic mean matter density at the present time.

\subsection{Model 2: model 1 + effect of primordial density field}

In the second variation of our model, we will relax the assumption that the temperature of the accreted material and the pre-shock density is the same for all structures, and we will consider the effect of variations in the local environment of accretors of the same mass caused by the structure of the primordial density field. In this case, accretors of the same mass will reside in a distribution of environments, parametrized by the local overdensity or underdensity (as determined by the evolution of primordial density fluctuations $), \delta_{\ell}=\left(\rho_{\text {local }}-\rho_{\mathrm{m}}\right) / \rho_{\mathrm{m}}$. In addition to affecting the pre-shock density enhancement, $\delta_{\mathrm{s}}+1=\delta_{\ell}+1$, such density variations will also cause temperature variations of the pre-shock gas, due to adiabatic heating or cooling. Since $c_{\mathrm{s}}^{2} \propto \rho_{\text {local }}^{\gamma-1}$, the pre-shock sound speed will in this case be a function of $\delta_{\ell}$,

$$
c_{\mathrm{s}}=c_{\mathrm{s}, \operatorname{avg}}\left(\delta_{\ell}+1\right)^{1 / 3},
$$

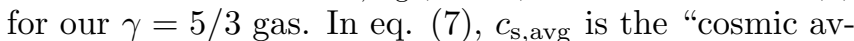
erage" sound speed (the sound speed of the intergalactic medium at a density equal to the cosmic mean at the epoch of interest), which we will again take to be equal to $15 \mathrm{~km} / \mathrm{s}$.

In order to make further progress and be able to calculate measures of the statistical properties of the population shocks in this approximation, we need an analytical model for the environment of collapsed structures. For this purpose, we will use the double distribution (DD) of collapsed structures with respect to mass and local overdensity (Pavlidou \& Fields 2005, hereafter PF05). In PF05, we defined the "local environment" of a collapsed structure through the clustering scale parameter, $\beta$. The clustering scale parameter is a free parameter in the double distribution model, and is defined so that the "environment" of an object of mass $m$ be a surrounding region in space which encompasses mass $\beta m$. Clearly, as $\beta \rightarrow 1$ the environment of an object is restricted to be closer to the object itself, and for $\beta=1$ it encompasses solely the collapsed object itself. Conversely, as $\beta \rightarrow \infty$, the environment of the object extends further and further away from the object, to eventually encompass the whole universe. 
With this definition of the "local environment", and using the same random walk formalism which yields the Press-Schechter mass function for collapsed objects, we found the DD to be

$$
\begin{aligned}
\frac{d n}{d m d \tilde{\delta}_{\ell}}= & \frac{\rho_{m, 0}}{m} \frac{\tilde{\delta}_{c}(a)-\tilde{\delta}_{\ell}}{2 \pi}\left|\frac{d S}{d m}\right|_{m} \exp \left[-\frac{\left(\tilde{\delta}_{c}(a)-\tilde{\delta}_{\ell}\right)^{2}}{2[S(m)-S(\beta m)]}\right] \\
& \times \frac{\exp \left[-\frac{\tilde{\delta}_{\ell}^{2}}{2 S(\beta m)}\right]-\exp \left[-\frac{\left(\tilde{\delta}_{\ell}-2 \tilde{\delta}_{c}(a)\right)^{2}}{2 S(\beta m)}\right]}{[S(\beta m)]^{1 / 2}[S(m)-S(\beta m)]^{3 / 2}}
\end{aligned}
$$

In Eq. (8), $S(m)$ is the variance of the linearly extrapolated overdensity field and $\tilde{\delta}_{\ell}$ is the local linearly extrapolated overdensity (or underdensity), which is related to the true (calculated from the spherical evolution model) overdensity $\hat{\delta}_{\ell}$ of the environment sphere including the local object through the exact relations given in PF05 or through the useful approximation (accurate at a better than $2 \%$ level throughout its domain for all cosmologies of interest)

$$
\tilde{\delta}_{\ell} \approx \frac{D\left(a_{0}\right)}{D(a)} \tilde{\delta}_{c}\left[1-\left(1+\hat{\delta}_{\ell}\right)^{-1 / \tilde{\delta}_{c}}\right],
$$

where $D(a)$ is the linear growth factor in the cosmology of interest and $\tilde{\delta}_{c}$ is the linear overdensity threshold for collapse in the same cosmology. The quantity $\hat{\delta}_{\ell}$ is in turn related to the overdensity of interest, $\delta_{\ell}$ (the overdensity of the environment sphere excluding the central object), through

$$
\delta_{\ell}=\frac{(\beta-1)\left(1+\hat{\delta}_{\ell}\right)\left(1+\delta_{c}\right)}{\beta\left(1+\delta_{c}\right)-\left(1+\hat{\delta}_{\ell}\right)}-1 .
$$

Because in the problem of cosmic accretion shocks we are interested in the properties (density and sound speed) of the material right outside the shock surface, we will adopt a small value for the clustering scale parameter, $\beta=1.1$. Our results, however, are not sensitive to the exact value of $\beta$ since, as we found in PF05, the properties of the double distribution (when calculated as a function of $\delta_{\ell}$ rather than $\hat{\delta}_{\ell}$ ) depend only mildly on $\beta$ for small values of $\beta$.

\subsection{Model 3 (full model): model $2+$ effect of filaments}

In this third variation of our model, we will expand the double-distribution-based model 2 to include the effect of filament preheating and compression. Most virialized structures reside inside filaments, and hence they are accreting gas which has already compressed and preheated in filament shocks. A complete description of the effect of filaments on the properties of cosmic accretion shocks would require an analytic model for the filamentary structure of the universe accounting for a distribution of different filament temperatures and densities, as well as their evolution with redshift, a problem which we will address in detail in an upcoming publication. Here, we adopt a simplified prescription which can provide a first assessment of the degree to which filaments alter the energetics and evolution of cosmic accretion shocks.
We will assume that all structures with environmental overdensity $\delta_{\ell}>0$ reside inside filaments ${ }^{3}$. These structures, instead of accreting gas of mean sound speed $c_{\mathrm{s} \text {,avg, }}$, will be accreting filament-preheated gas of mean sound speed $c_{\mathrm{s}, \text { fil }} \approx 45 \mathrm{~km} / \mathrm{s}$, corresponding to a (conservative) mean filament temperature $T_{\text {fil }} \approx 10^{5} \mathrm{~K}$ (e.g. Cen \& Ostriker 1999, Davé et al. 2001; Fang et al. 2002). In addition, $1+\delta_{\ell}$ will now represent the compression factor with respect to a mean filament density $\rho_{\text {fil }}=\rho_{\mathrm{b}}\left(1+\delta_{\text {fil }}\right)$ where (again, conservatively $)^{4} 1+\delta_{\text {fil }} \approx 10$. Hence, in model 3 the pre-shock sound speed will be

$$
c_{\mathrm{s}}=\left\{\begin{array}{ll}
c_{\mathrm{s}, \text { avg }}\left(\delta_{\ell}+1\right)^{1 . / 3}, & \delta_{\ell} \leq 0 \\
c_{\mathrm{s}, \text { fil }}\left(\delta_{\ell}+1\right)^{1 . / 3}, & \delta_{\ell}>0
\end{array},\right.
$$

while the pre-shock density enhancement will be

$$
1+\delta_{\mathrm{s}}=\left\{\begin{array}{ll}
1+\delta_{\ell}, & \delta_{\ell} \leq 0 \\
\left(1+\delta_{\mathrm{fil}}\right)\left(1+\delta_{\ell}\right), & \delta_{\ell}>0
\end{array} .\right.
$$

The distribution of number density of accretors with respect to mass and local overdensity $\delta_{\ell}$ will again be assumed to be well described by the double distribution of collapsed structures.

\subsection{Quantitative measures of the properties of cosmic accretion shocks}

The specific properties of the population of cosmic accretion shocks which we wish to investigate can be classified into two categories. First of all, we are interested to see how the shock-processed mass and energy is distributed among shocks of different Mach numbers, and how environmental effects alter this distribution. Such Mach number distributions are of particular importance to assess the role of cosmic accretion shocks as potential sites of particle acceleration, since the highestenergy particles can be accelerated only in the strongest $(\mathcal{M} \gg 1)$ shocks. The quantities we will calculate to address this question are:

- The "mass current distribution" with respect to Mach number, $d J / d \ln \mathcal{M}$, defined as the comoving mass current density crossing shock surfaces of logarithmic Mach number between $\ln \mathcal{M}$ and $\ln \mathcal{M}+d \ln \mathcal{M}$, with units of $\mathrm{M}_{\odot} \mathrm{yr}^{-1} \mathrm{Mpc}^{-3}$. In the case of model 1 , it is given by

$$
\frac{d J}{d \ln \mathcal{M}}=\left.\mathcal{M} \frac{d n}{d m} \frac{\partial m}{\partial \mathcal{M}}\right|_{z} J_{1},
$$

3 This is a conservative assumption. In reality, even structures predicted by the double distribution model to reside inside underdensities may be found inside filaments, as neighboring voids may sufficiently compress their surrounding regions.

4 Note that our prescription does not account for filament evolution with cosmic time. Of course, filaments do evolve with redshift, and a more detailed calculation, based on a model accounting for filament evolution as well as different filament overdensities and temperatures is desirable and currently pursued. However, the error introduced by not accounting for filament evolution is expected to be relatively small, as filaments primarily evolve by shock-heating and compressing increasing quantities of diffuse gas, rather than heating it and compressing it to significantly different densities and temperatures (see e.g. distributions in Davé et al. 2001, which change in amplitude rather than shift in location with changing redshift). This however is not expected to significantly affect the properties of gas accreted by virialized structures: filaments may include smaller quantities of gas at high redshifts, but structures are also smaller in size and most of them are still expected to be found inside filaments. 
where $m=m(\mathcal{M}, z)$ is given by eqs. (1) and (2) with $c_{\mathrm{s} 1}=15 \mathrm{~km} / \mathrm{s} ; d n / d m$ is the Press-Schecter mass function, given by eq. (6); and $J_{1}$ is given by eq. (3) with $\delta_{\mathrm{s}}=0$. In the case of models 2 and 3, it is given by

$$
\frac{d J}{d \ln \mathcal{M}}=\left.\mathcal{M} \int_{\delta_{\ell}=-1}^{\delta_{c}} d \delta_{\ell} J_{1} \frac{d n}{d m d \delta_{\ell}} \frac{\partial m}{\partial \mathcal{M}}\right|_{\delta_{\ell}, z},
$$

where $d n /\left(d m d \delta_{\ell}\right)$ is the double distribution of cosmic structures, given by eq. (8); $m(\mathcal{M}, \delta, z)$ is again given by eqs. (11) and (2), with $c_{\mathrm{s} 1}$ given by eqs. (71) and (11) for models 2 and 3 respectively; and $\delta_{\mathrm{s}}=\delta_{\ell}$ for model 2 , while $\delta_{\mathrm{s}}$ given by eq. (12) for model 3 .

- The "kinetic power distribution" with respect to Mach number, $d P / d \ln \mathcal{M}$, defined as the comoving kinetic power density crossing shock surfaces of logarithmic Mach number between $\ln \mathcal{M}$ and $\ln \mathcal{M}+d \ln \mathcal{M}$, with units $\operatorname{erg~s}^{-1} \mathrm{Mpc}^{-3}$. It is given by

$$
\frac{d P}{d \ln \mathcal{M}}=\left.\mathcal{M} \frac{d n}{d m} \frac{\partial m}{\partial \mathcal{M}}\right|_{z} P_{1}
$$

for model 1 and by

$$
\frac{d P}{d \ln \mathcal{M}}=\left.\mathcal{M} \int_{\delta_{\ell}=-1}^{\delta_{c}} d \delta_{\ell} P_{1} \frac{d n}{d m d \delta_{\ell}} \frac{\partial m}{\partial \mathcal{M}}\right|_{\delta_{\ell}, z},
$$

for models 2 and 3 . In both cases, $P_{1}(\mathcal{M}, z)$ is given by Eq. (5).

Second, we would like to follow the cosmic evolution of the energetics of cosmic accretion shocks, tracing their mass and energy processing and their impact on the intergalactic medium. In particular, we wish to calculate the energy amount processed by all cosmic accretion shocks per unit time and by a certain redshift, as well as the degree to which cosmic baryons have been affected by cosmic accretion shocks by some specific cosmic epoch. The quantities we will use to quantify these questions are:

- The "integrated mass current density", $J$, which is the comoving mass current density crossing shock surfaces of any Mach number at a given cosmic epoch, with units of $\mathrm{M}_{\odot} \mathrm{yr}^{-1} \mathrm{Mpc}^{-3}$.

- The "integrated kinetic power density", $P$, which is the comoving kinetic power density crossing shock surfaces of any Mach number at a given cosmic epoch, with units of $\operatorname{erg~s}^{-1} \mathrm{Mpc}^{-3}$.

- The "cumulative processed kinetic energy density", $\int_{t_{\mathrm{i}}}^{t} P d t$, which is the total kinetic energy density processed by shocks of any Mach number since some initial cosmic epoch $t_{\mathrm{i}}$, with units of $\mathrm{eV}$ per baryon in the universe.

The second set of quantities will be calculated as a function of redshift, and for each of the models 1, 2 and 3, in order to assess the effect of each environmental factor on their behavior.

\section{RESULTS}

Throughout this section we will assume a flat, vacuum+matter universe with Wilkinson Microwave Anisotropy Probe (WMAP) parameters $\sigma_{8}=0.84, h=$ $0.71, \Omega_{\mathrm{m}}=0.27$ and $\Omega_{\mathrm{b}}=0.04$ (Spergel et al. 2003). We focus on post-reionization redshifts, hence we adopt a cosmic average sound speed of $15 \mathrm{~km} / \mathrm{s}$, corresponding to a temperature of $\sim 10^{4} \mathrm{~K}$, for a fully ionized plasma with $\mu=0.59$ ( $25 \%$ He by mass).

\subsection{Mach number distributions and the effect of the environment}

The distributions with respect to Mach number of mass current and kinetic power are plotted in figs. 11 and 2 respectively. In both figures, the solid line corresponds to model 1 (Press-Schechter-based, no environmental effects); the dashed line corresponds to model 2 (doubledistribution-based, includes the effect of the primordial density field but not the effect of filaments); and the dot-dashed line corresponds to model 3 (includes effects of both primordial density field as well as effects of filaments). The left panel shows the results for $z=3$, while the right panel corresponds to the present-day universe.

The results of the Press-Schecter-based, noenvironmental-effects model can be interpreted in a straight-forward way. In this case, accretor mass and Mach number have a one-to-one correspondence, with larger accretor masses resulting to higher-Mach-number accretion shocks. As cosmic time progresses, the mass of the largest collapsed objects increases, as more and more massive structures have had enough time to collapse. Hence, the strongest accretion shocks become stronger with decreasing redshift, and the distributions of mass current and kinetic energy move to higher Mach numbers with increasing time. The kinetic power distribution is more strongly suppressed at low Mach numbers (and therefore low accretor masses) than the mass current distribution. This result can be immediately verified by simple analytic arguments. For masses high enough that the primordial density fluctuation power spectrum can be regarded as a power law but low enough that the exponential mass cutoff is not affecting the results, $d n / d m \propto m^{-2}$. In addition, $\mathcal{M} \propto m^{1 / 3}$ (in the high- $\mathcal{M}$ limit), while $r_{\mathrm{v}} \propto m^{1 / 3}$. Hence, $J_{1} \propto m$ and $P_{1} \propto m^{5 / 3}$. Equations (13) and (15) then give for the low-mass dependence of the mass current and kinetic power distributions,

$$
\begin{aligned}
& \frac{d J}{d \ln \mathcal{M}} \propto m^{0} \propto \mathcal{M}^{0} \\
& \frac{d P}{d \ln \mathcal{M}} \propto m^{2 / 3} \propto \mathcal{M}^{2}
\end{aligned}
$$

accounting for the difference in the low-mass behavior of different distributions. Note that the low-mass suppression is somewhat stronger than what predicted by the simple arguments above, due to the deviation of $\mathcal{M}(m)$ from $m^{1 / 3}$ for $\mathcal{M} \rightarrow 1$.

It should be pointed out that the result that it is the highest-Mach-number shocks that process most energy refers to the energy processed by accretion shocks alone (which are the shocks treated in this work). Merger shocks are generally expected to dominate the overall energetics of cosmic shocks (e.g. Miniati et al. 2000; Gabici 

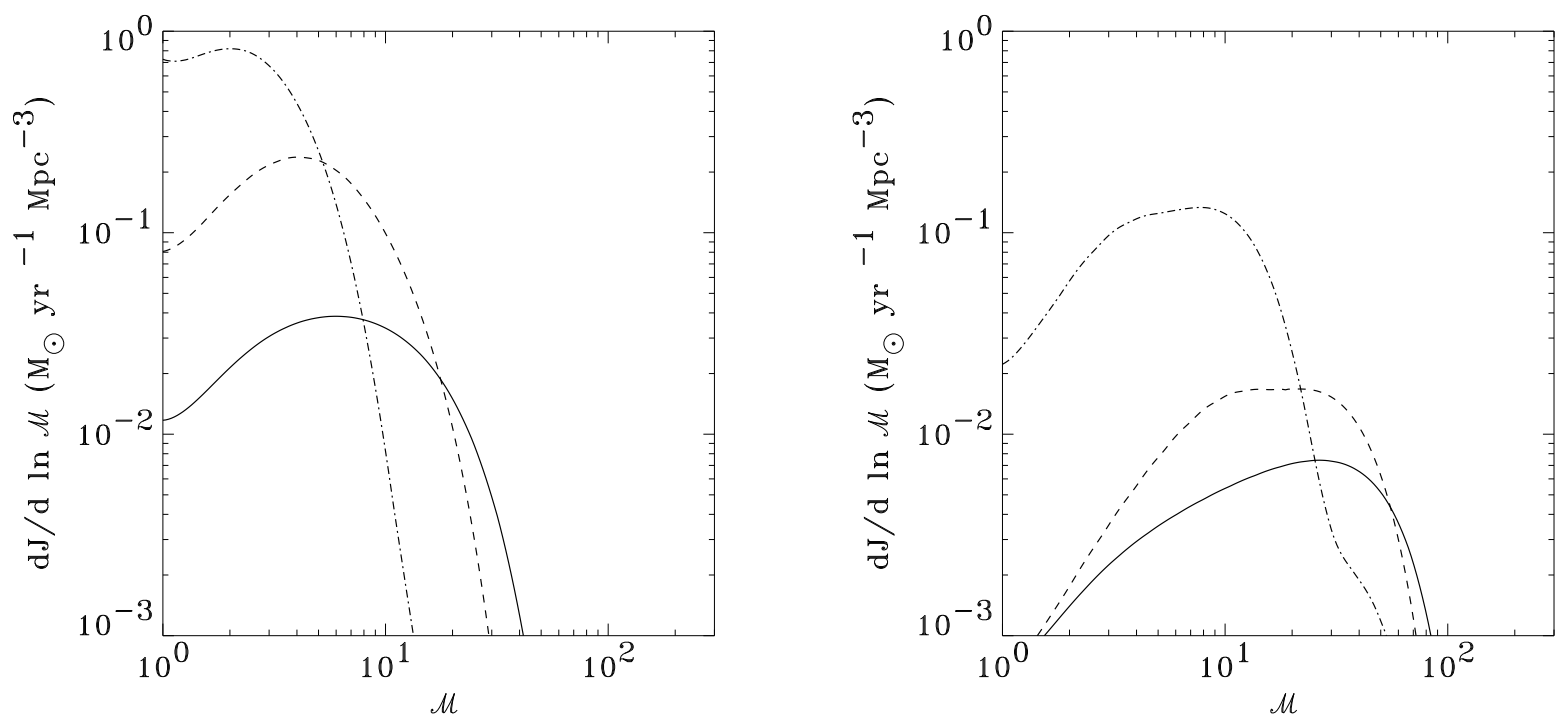

FIG. 1.- Mass current distribution (spatial density of mass accretion rate per logarithmic Mach number interval, $d J / d \ln \mathcal{M}$ )for $z=3$ (left panel) and $z=0$ (right panel). The units of the vertical axes are $\mathrm{M}_{\odot} \mathrm{yr}^{-1}$ comoving $\mathrm{Mpc}^{-3}$. Solid line: model 1 (no environmental effects); dashed line: model 2 (effect of primordial density fluctuations); dot-dashed line: model 3 (effects of primordial density fluctuations and filaments).
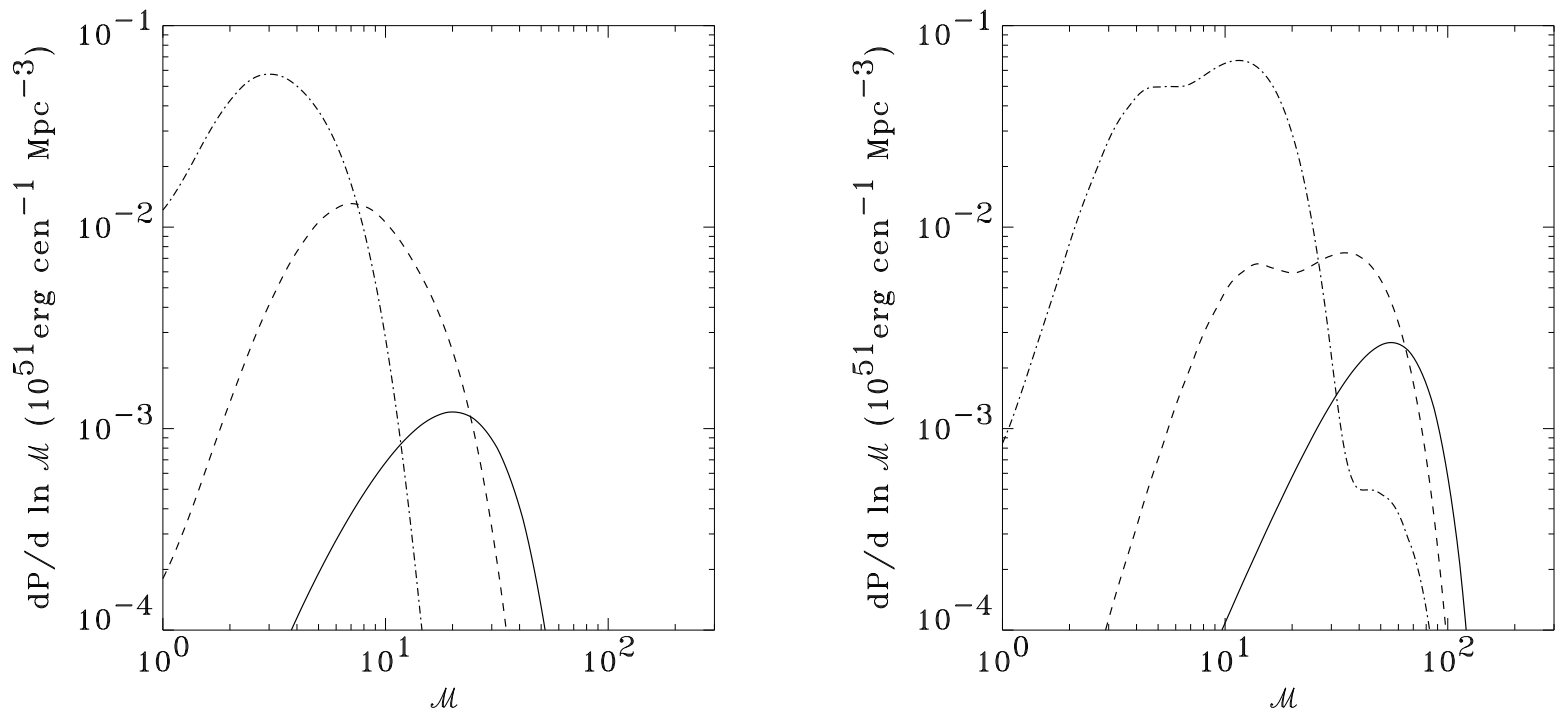

FIG. 2.- Kinetic power distribution (spatial density of kinetic power processed by accretion shocks per logarithmic Mach number interval, $d P / d \ln \mathcal{M}$ )for $z=3$ (left panel) and $z=0$ (right panel). The units of the vertical axes are $\mathrm{g} \mathrm{s}^{-1}$ comoving Mpc $^{-3}$. Solid line: model 1 (no environmental effects); dashed line: model 2 (effect of primordial density fluctuations); dot-dashed line: model 3 (effects of primordial density fluctuations and filaments).

\& Blasi 2003; Ryu et al. 2003), because the density of the (already virialized) gas they process is much higher (by a factor between $\sim 20$ and 200 on average, depending on the environment in which accretion shocks lie) than the density of the gas processed by accretion shocks. However, because the virialized gas relevant to merger shocks has also been preheated to much higher temperatures compared to the gas accreted by accretion shocks, the Mach numbers associated with merger shocks are significantly lower. Hence, accretion shocks are expected to dominate the strong shock energetics.

The effect of taking into account the environmental density fluctuations in the primordial density field using the double distribution of collapsed structures is demonstrated by the curves corresponding to model 2 , and is two-fold. On the one hand, every mass bin is spread out to a larger $\mathcal{M}$ range, as each accretor mass now corresponds to a distribution of Mach numbers, reflecting the associated distribution of environmental overdensities. On the other hand, the local density of the material immediately outside the accretor is now correlated with the accretor mass. Higher-mass objects, which have a dominant contribution to the kinetic power and, to a lesser extent, the mass processed by accretion shocks, 
tend to reside in higher-mass environments. As a result, the overall amplitude of both the kinetic power and the mass current distribution is increased.

A most striking environmental effect in the case of the kinetic power distribution is that, as redshift decreases, a second peak separates out in model 2, which is absent in the base model. The presence of this second, high- $\mathcal{M}$ peak is due to the gradual shift of increasingly massive structures towards underdense environments. The lower$\mathcal{M}$ peak is, conversely, due to the higher-mass structures which are embedded inside higher-overdensity and hence higher-temperature environments.

The additional effect of the filamentary structure of the universe is demonstrated by the curves corresponding to model 3. The inclusion of filaments results to a shifting of both the mass current and the kinetic power distributions towards lower Mach numbers (due to the preheating of the pre-shock gas by filament shocks) and higher amplitude (due to the additional density enhancement of pre-shock material inside filaments). At low redshifts, the amplitude of both distributions shifts by an order of magnitude, reflecting our assumed mean filament overdensity. Although our recipe for the inclusion of the effect of filaments in model 3 is only a first approximation to the analytical treatment of the issue, there are two robust conclusions we already draw from this analysis. First, the effect of filaments is at least of the magnitude derived here, since all of our assumptions were chosen so that they err on the side of underestimating both the filament-associated preheating as well as density enhancement. Hence, our results emphasize the need for a detailed model for filament-associated processes and their cosmic evolution, which have been shown to be an important factor in determining the statistical properties of cosmic accretion shocks. Second, the direction of the changes in the mass current and kinetic power distributions due to filaments will be towards lower Mach numbers and higher amplitudes, if anything at a higher degree than predicted here. Even with more sophisticated models, this result is not expected to change qualitatively.

Note that the mean filament temperature we have used $\left(T \sim 10^{5} \mathrm{~K}\right)$ is rather on the low-end of the temperature distribution of filaments in the present-day universe as found in numerical simulations. Since we do not account for redshift evolution of the mean filament temperature, we have chosen this low value to ensure that we do not overestimate the effect of filament preheating on accretion shocks at higher redshifts. A more realistic mean filament temperature of $T \sim 10^{6} \mathrm{~K}$ at low redshifts (right panels of Figs. 1 and 2) would roughly result to a horizontal translation of the model 3 curve towards lower Mach numbers by a factor of $\sim 3$.

\subsection{Integrated quantities and evolution with cosmic epoch}

Figure 3 shows the evolution of the integrated mass current $J$ (left panel) and integrated kinetic power $P$ (right panel) for shocks of any Mach number, and for redshifts between 6 and 0 . The solid line corresponds to model 1 , the dashed line to model 2 , and the dotdashed line to the "full" model 3 . The thick solid line corresponds to the supernova energy input to the intergalactic medium (which is discussed in detail in the next section).

The integrated mass current peaks at $z \sim 2$ in the case of model 3 , while the models 1 and 2 mass current histories peak closer to $z \sim 3$. The reason for this difference is that the filament preheating and the associated decrease of accretion shock Mach number for given accretor mass result to a certain loss of power from the mass current distribution, as the lowest mass objects can no longer satisfy $\mathcal{M}>1$. The effect is more pronounced at higher redshifts, when the largest collapsed objects are less massive than the largest objects in the local universe. As a result, the evolution of the mass current is more severe at high redshifts for model 3 , and the integrated mass current peaks at a lower redshift than in the other two models.

The same effect is present but less pronounced in the case of the integrated kinetic power: the high-z tail of model 3 falls more sharply with increasing redshift than the tail of models 1 and 2, however the effect is not strong enough to affect the location of the peak, which is found at $z \sim 1$ for all three models.

The difference in the location of the peak in redshift between integrated mass current and kinetic power is due to the difference in the mass dependence of the associated distributions. The kinetic power distribution depends on mass much more strongly than the mass current, and thus the integrated kinetic power history "prefers" lower redshifts, where the largest collapsed structures (which process most of the kinetic power crossing accretion shocks) are more massive. For the same reason, the "loss of power" due to the presence of filaments is much milder in the kinetic power history: the lower-mass structures, which are in danger of "dropping out" from the population of structures hosting shocks in a highertemperature environment, do not contribute much to the kinetic power distribution to begin with.

At redshifts close to their respective peaks, the integrated mass current and kinetic power as calculated by model 2 are enhanced by about an order of magnitude with respect to the no-environmental-effects model 1. Similarly, the predictions of model 3 (filaments + primordial overdensities) are about an order of magnitude enhanced with respect to those of model 2 (primordial overdensities only).

\subsection{Comparison of accretion shocks with other intergalactic medium energy inputs}

In this section, we will discuss how the kinetic energy processed by cosmic accretion shocks compares with other energy inputs and characteristic energy scales of the intergalactic medium.

In the right panel of fig. 3 we have plotted, along with the integrated kinetic power of cosmic accretion shocks as predicted by models 1,2 and 3, the energy input of type II supernovae as a function of redshift (thick solid line). The supernova energy history was derived from the Cole at al (2001) fit to their dust-corrected measurements of the cosmic star formation rate, assuming a Salpeter mass function with a supernova progenitor mass cutoff of $8 \mathrm{M}_{\odot}$, and adopting a supernova explosion energy of $10^{51} \mathrm{erg}$. The accretion-shock-processed energy is higher than the energy output of supernovae for all redshifts $\lesssim 3$. In addition, at low redshifts the star formation rate decreases with decreasing redshift much more strongly 

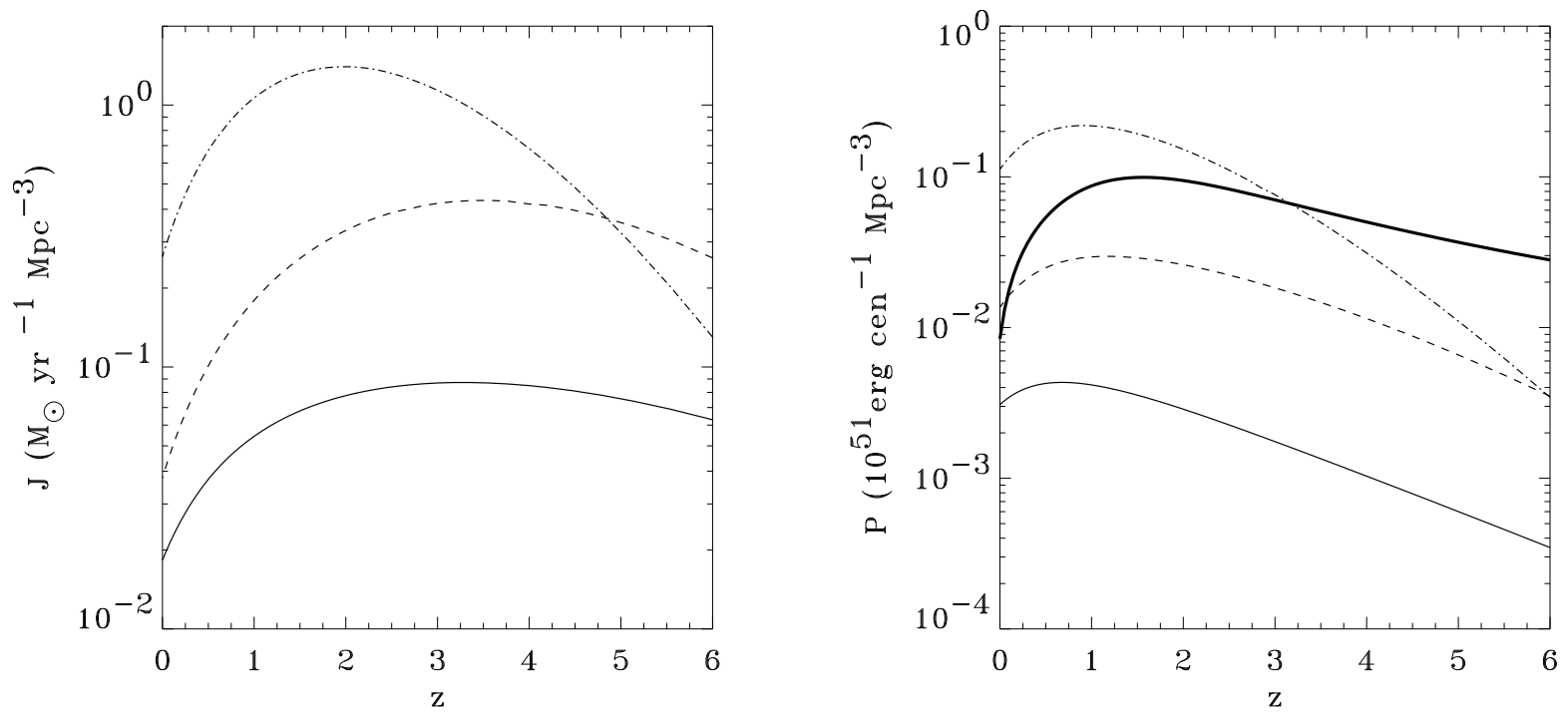

FIG. 3.- Integrated mass current $J$ (left panel) and integrated kinetic power $P$ (right panel) as a function of redshift. Solid line: model 1 (no environmental effects); dashed line: model 2 (effect of primordial density fluctuations); dot-dashed line: model 3 (effects of primordial density fluctuations and filaments). The thick solid line in the right panel represents the energy output of supernovae as derived from the Cole et al. (2001) fit for the cosmic star formation rate.

than the accretion shock processed power, and as a result in the local universe the energy input to the intergalactic medium due to accretion shocks is expected to be (at least) one order of magnitude higher than the energy output of supernovae.

¿From the point of view of particle acceleration, despite the fact that the efficiency with which accretion shocks may accelerate high-energy particles is largely unconstrained, the energy available for particle acceleration is much larger than in the case of supernovae, and in this respect the potential of cosmic accretion shocks as sites of cosmic ray acceleration is once again seen to be promising.

Finally, in Fig. 4 we plot the cosmic history of $\int P d t$ (the cumulative processed kinetic energy) in units of eV per baryon in the universe (as opposed to per shocked baryon). Again, the solid line corresponds to model 1, the dashed line to model 2 and the dot-dashed line to the "full" model 3. The horizontal line in this plot corresponds to $13.6 \mathrm{eV}$ per baryon. ¿From the location of the intersection of the horizontal line with the $\int P d t$ curve, we can conclude that by redshift $z \sim 3.5$ (for model 3 ), the energy processed by accretion shocks alone would have become comparable to the energy needed to reionize the universe ${ }^{5}$.

\section{COMPARISON TO PAST WORK}

\subsection{Comparison to baryon energetics from cosmological simulations}

If we integrate $J$ (the mass current through shocks of any Mach number) as predicted by model 3 (dot-dashed line in left panel of Fig. 3) over time, we find that by

5 This is an order-of-magnitude estimate, meant to give a feeling of the amount of energy processed by shocks as compared to other energy inputs in the IGM. If one wanted to consider shocks as an actual reionization mechanism, a detailed modeling of the reionization process would be required, see e.g. Miniati et al. 2004.

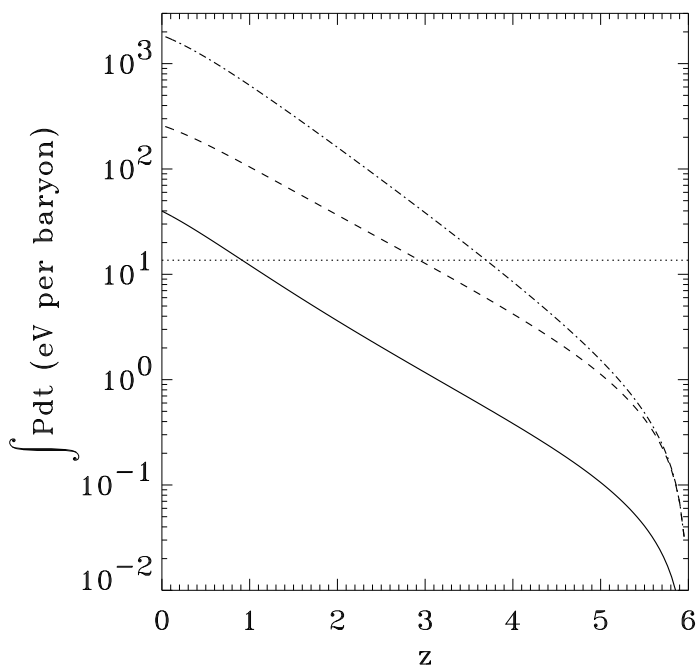

FIG. 4.- Cumulative kinetic power $\int P d t$ (in units of eV per baryon in the universe), as a function of redshift. Solid line: model 1; dashed line: model 2; dot-dashed line: model 3. The horizontal dotted line corresponds to $13.6 \mathrm{eV}$ per baryon.

the present cosmic epoch, $51 \%$ of the baryons in the universe have been processed by accretion shocks, and are therefore residing within structures of virial temperature roughly $>10^{4} \mathrm{~K}$ (mass $\left.\gtrsim 10^{10} \mathrm{M}_{\odot}\right)$. We can compare this value to the findings of cosmological simulations. If we assume that $1 / 2$ of the baryons of temperatures between $10^{4}-10^{7} \mathrm{~K}$ belong to collapsed objects of corresponding virial temperatures, and we add these baryons to the "condensed" (cooled and belonging to galaxies) and "hot" $\left(T>10^{7} \mathrm{~K}\right.$ and belonging to high-mass collapsed objects) baryons, we get a total baryon fraction which is between $35-60 \%$, depending on the specifics of each 
simulation (see, e.g. simulations in Davé et al. 2001).

As far as the cumulative energy processed by accretion shocks $\int P d t$ is concerned, the prediction of model 3 for by $z=0$ is $P \approx 2 \mathrm{keV}$ per baryon in the universe (dotdashed line in Fig. (4). Note that this is not a prediction for the mean per baryon in the universe energy today, since our calculation does not account for any cooling losses or any other energy inputs to the IGM. Still, it is interesting to note that it is within a factor of $\sim 2$ of the value found in cosmological simulations by e.g. Cen \& Ostriker (1999), who (accounting for cooling as well as star formation feedback) calculate a density-weighted average temperature (the quantity representing the mean per-baryon-in-the-universe energy) of $\sim 10^{7} \mathrm{~K}$ at $z=0$.

It is therefore very encouraging to see that, given the simplifying assumptions and idealizations of our model (spherically symmetric infall, Press-Schechter mass function, absence of time evolution in filament temperature and density), our results are in reasonable agreement with the findings of cosmological simulations, indicating that our most sophisticated model (model 3) has captured the essential elements of the gravity-driven, diffuse-matter-accretion-associated energetics of cosmic baryons. More importantly, we see that our other two models, which differ 1-2 orders of magnitude in their predictions for the cumulative quantities discussed above, are not sufficient to reproduce the results of cosmological simulations. This fact is suggestive of the importance of filaments not only in processing baryons in their own filament shocks and giving rise to the warm-hot intergalactic medium, but also in modifying the energetics of accretion shocks.

\subsection{Comparison to simulations of cosmic shocks}

Figure 5 shows two quantities commonly used in the cosmic shock literature to describe the properties of populations of different types of cosmic shocks. In the left panel, we plot the distribution of the comoving number density of accreting structures per logarithmic Mach number interval of their respective accretion shocks, $d n / d \ln \mathcal{M}$, with units number of structures per comoving $\mathrm{Mpc}^{3}$, for the three models we have presented in this work and for $z=0$. In the right panel, we plot the distribution of the comoving shock surface area per logarithmic Mach number interval per volume under consideration, $d S / d \ln \mathcal{M}$ with units of $\mathrm{Mpc}^{-1}$ (ratio of shock surface over space volume). Only structures with virial temperatures $>10^{4} \mathrm{~K}\left(\right.$ masses $\left.>8 \times 10^{9} \mathrm{M}_{\odot}\right)$ are plotted. As expected, these distributions are dominated by the lowmass, low-Mach-number structures (the same analytic scalings used in 4.1 give, for the low-mass behavor of our no-environmental-effects model $1 d n / d \ln \mathcal{M} \propto m^{-1}$ and $\left.d S / d \ln \mathcal{M} \propto m^{-1 / 3}\right)$. The number distribution in the Press-Schechter-based model (model 1) monotonically increases for decreasing $\mathcal{M}$ since in this case there is a one-to-one correspondence between mass and Mach number. Hence the number distribution of objects simply follows the Press-Schechetr mass function modulated by the mass-Mach conversion. In the case of the double-distribution-based models (models 2 and 3 ), the mass cutoff, in combination with the distribution of preshock sound speeds for each accretor mass described by the double distribution, results in a number distribution which peaks at $\mathcal{M} \lesssim 4$, a position which is defined by the combination of the virial temperature at the mass cutoff (which is the most populated available mass bin) and the sound speed corresponding to the most probable environment at that particular mass. Note that the difference in both the number and surface distributions between models 2 and 3 (without and with the effect of filaments) is very small. This is because we have used the double distribution of cosmic structures to predict which structures are located inside filaments, and the double distribution predicts that most of the low-mass cosmic structures (the ones dominating the number and surface distributions) are found inside underdense regions, and hence not inside filaments.

For the case of accretion shocks which we have studied here, we can then draw the following conclusions:

1. The energetics of these shocks are determined by the few, high-Mach-number, high-mass structures, and are not significantly affected by the majority of structures, which have low masses and low accretion shock Mach numbers.

2. Our results are in natural agreement with the findings of Ryu et al. (2003), who performed a series of simulations with increasing mass and spatial resolution, and found convergence for their results on kinetic power distribution (dominated by highmass structures) but not for the surface distribution (dominated by low-mass structures). However, since the physical impact of shocks to their environment is better represented by the kinetic power distribution, which is not sensitive to the behavior of low-mass structures, the lack of convergence in the number-dominated distributions in cosmological simulations should not decrease confidence in the relevance and robustness of the conclusions of such cosmological simulations relating to the effect of accretion shock on the intergalactic medium.

Finally, we should caution the reader that a direct comparison between our Fig. (5) and similar plots in cosmic shock literature is not straight-forward - the shocks treated here are exclusively accretion shocks, and they do not include merger or filament shocks. When shock surfaces in the universe are labeled as internal or external, accretion shocks are split between the two types: internal shocks can be merger shocks, or they can be accretion shocks associated with structures found inside filaments; external shocks on the other hand can be accretion shocks associated with structures which are not located inside filaments, or they can be filament shocks.

\subsection{Comparison to other analytic models of cosmic shock populations}

Press-Schechter extensions have been used extensively to model different "families" of large-scale cosmic shocks, with emphasis primarily on the emission properties of such populations, rather than the properties of the shocks themselves.

Waxman \& Loeb (2000) assumed a Press-Schechter mass function to describe an underlying population of accretors hosting particle accelerating shocks which would produce, through their radio and gamma emission, fluctuations in the radio and gamma-ray background. All 

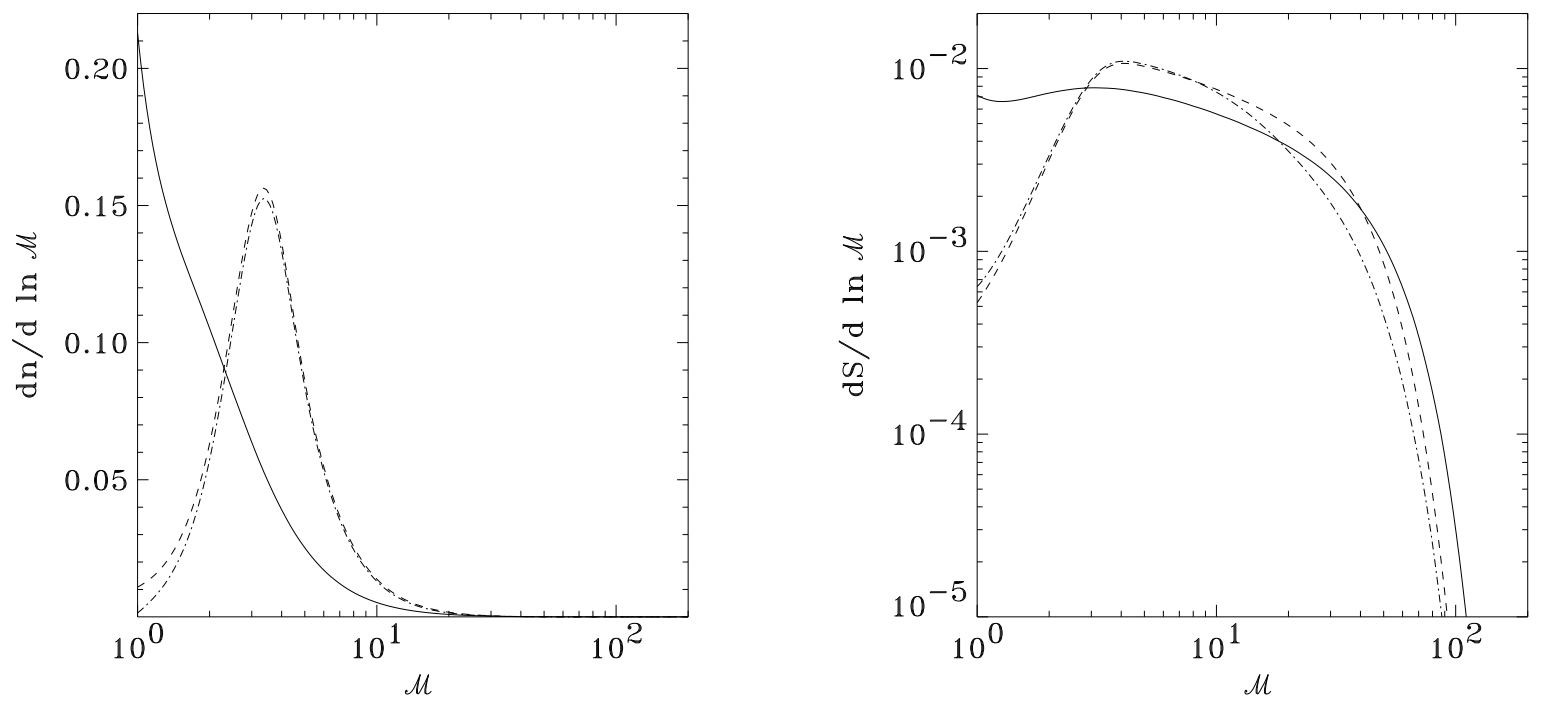

FIG. 5.- Left panel: distribution of the comoving number density of accreting structures per logarithmic Mach number interval of their respective accretion shocks, $d n / d \ln \mathcal{M}$, with units number of structures per comoving $\mathrm{Mpc}^{3}$. Right panel: distribution of the comoving shock surface area per logarithmic Mach number interval per volume under consideration, $d S / d \ln \mathcal{M}$ with units of $\mathrm{Mpc}^{-1}$. Solid line: model 1; dashed line: model 2; dot-dashed line: model 3. All curves correspond to $z=0$. Only structures with masses $>8 \times 10^{9} \mathrm{M}_{\odot}$ are plotted.

shock quantities required for their emissivity and fluctuation calculations (mass accretion rate, shock temperature, shock radius) were derived as functions of the accretor velocity dispersion up to dimensionless constants, left to be calibrated against simulations. Of all the analytic shock models discussed in this section, the Waxman \& Loeb (2000) model is the one with the most similarities with the "base" model we use in this work (the model containing no environmental effects). The two primary similarities are that a) we also take the underlying accretor distribution to be described by a Press-Schechter mass function and b) the mass-scaling of the shock properties is the same over a large range of masses. In our case, the shock properties are obtained the temperature jump across the shock, which involves the massdependent virial temperature of each object and some environmental temperature. At the large-temperaturejump/high-Mach-number limit, the mass dependencies of shock properties in our model agree with those of Waxman \& Loeb (2000), as the velocity dispersion and the free-fall velocity around an object of a certain mass (which is encoded in the accretion shock Mach number provided that Mach $\gg 1$, or, equivalently, that the gas pressure effects in the pre-shock gas are negligible) necessarily have the same mass dependence.

However, our "base model" describes different aspects of the accretion shock population that Waxman \& Loeb (2000), as they calculate the shock gamma and radio emission properties while we calculate the mass current and shock energetics, exhibit their dependence on Mach number, calculate the cosmic distribution of mass and energy flows as a function of Mach number, and explicitly construct histories for the accreted mass and energy.

Fujita \& Sarazin (2001) used a version of the Lacey \& Cole (1993) merger formalism (a Press-Schechter extension) to describe mergers and accretion in a unified way (in their picture, accretion is the sum of all minor mergers for which the relative mass increment of the accreting halo does not exceed some threshold). They as well use their model to obtain the nonthermal emission properties of merging and accreting clusters, and they compare how such properties differ between clusters which have recently undergone some major merger and clusters which have been only accreting through minor mergers. Treating accretion as a subcase of the general merger picture associated with hierarchical structure formation is a widely used technique. It was adopted also by Gabici \& Blasi (2003a, b) who also used a semi-analytic model based on the Lacey \& Cole (1993) merger formalism to calculate the Mach numbers of shocks resulting from mergers, the particle acceleration properties of such shocks, and the contribution of merger-shock-accelerated particles to nonthermal radiation from large-scale structure (including cluster radio halos and a contribution to the gamma-ray background $)^{6}$.

Such a mergers-only approach is fundamentally rather than just formally different than the one we use here. First of all, since in the mergers-only picture all matter is locked up in collapsed objects of various masses, the pre-shock material has a mixture of temperatures, characteristic of the virial temperatures (and hence masses) of the accumulated halos, rather than of the diffuse environment of the object. In addition, the mass accretion rate for a halo of mass $m$ in the mergers-only picture is not $\propto m$ as in the simple, gravitationally-driven accretion of diffuse gas picture we adopt, but is dependent on the index $n$ of the power spectrum of initial perturbations

\footnotetext{
${ }^{6}$ Note however that Gabici \& Blasi (2004) did make an explicit distinction between mergers and diffuse gas accretion when assessing the detectability of gamma rays from galaxy clusters; in this work, they considered all accretion shocks to have $\mathcal{M} \gg 1$, while the scaling of shock energy with accretor mass was similar to that of Waxman \& Loeb (2000).
} 
(and is $\propto m^{7 / 6}$ for $n=1$ ). This difference stems from the different physical processes followed in each case. In the mergers-only, Press-Schechter-extension picture, growth of structure, whether through accretion or through mergers, occurs due to the gradual turnaround and collapse of increasingly large scales. This process is caused by the gravitational enhancement of perturbations already imprinted in the primordial density field. The gravitational effect of a collapsed object within such a larger collapsing structure is simply to contribute to the mean density of the collapsing region. In our picture, accretion is driven locally, through the gravitational attraction that a collapsed structure exerts on its surrounding diffuse gas. Each collapsed structure would accrete gas even if it lived inside an otherwise homogeneous universe. In nature, both processes contribute to the growth of any particular collapsed structure, and it is hence important to construct analytic models based on both pictures, and gauge the relevant importance of each process through comparison with observations and simulations.

More recently, Inoue \& Nagashima (2005) used the Somerville \& Kolatt (1999) multiple merger model to follow the population of cosmic shocks, calculate the associated gamma-ray emission and investigate the possibility to use gamma-ray observations as a tool for the study of the warm-hot intergalactic medium. The Somerville \& Kolatt (1999) merger model is an improved version of a Press-Schechter-based semi-analytic merger-tree construction algorithm. Its major advantage with respect to other algorithms for construction of merger trees of present-day objects is that it predicts a distribution of mass progenitors which is consistent with the extended Press-Schechter predictions while it enforces strict mass conservation. This model accounts for some amount of diffuse accreted matter, which is however calculated not by gravitational arguments, but again as that amount of matter corresponding to progenitors less massive than some pre-set cutoff.

Finally, Furnaletto \& Loeb (2004) used a modified Press-Schechter formalism to describe large-scale shocks that may appear when overdense perturbations reach and exceed their turnaround point. The shocks they consider are located at the boundaries between regions which have reached their turnaround point, and the background (still expanding) universe. The shock velocity is derived from a scaling argument to be approximately equal to the size the shocked region would have had if it had expanded with the Hubble flow, divided by the age of the universe. All shocks in this work are assumed to be strong, and Mach-number dependencies are not investigated. ¿From a physical standpoint, the shocks described by these authors occur much earlier in the evolution of each structure than the accretion shocks we describe here, and they are the shocks responsible for heating up the bulk baryonic component of collapsed objects from mean intergalactic medium temperatures to the virial temperature of structures. In our shock classification scheme, the Furlanetto \& Loeb (2004) shocks would be the gravitationally-driven component of filament shocks.

To summarize, our model of accretion shocks is different from and complementary to all of the exiting treatments. Our focus on the role of diffuse gas accretion is qualitatively and quantitatively different from the mergers-only approaches, and these differences can be exploited to test against the physical reality in which both accretion modes occur. Our model is most similar to that of Waxman \& Loeb (2000), but we extend the Press-Schechter treatment to include both the effects of environment, and to address the effects of preheating by filament shocks. Finally, our work differs from much of the existing literature by focusing on the shock energetics, and to our knowledge is the first to explicitly calculate the net cosmic processing of both energy and mass through accretion shocks over cosmic history.

\section{DISCUSSION}

In this paper, we have investigated analytically the properties of cosmic accretion shocks around collapsed structures. We have calculated the mass and kinetic energy currents as a function of Mach number for accretion shocks on individual structures of arbitrary mass at any epoch. Using this, we have computed the cosmic distribution of mass and energy flows as a function of Mach number, and we have calculated the evolution of these quantities as a function of redshift.

We have found that environmental factors play a major role in shaping the qualitative and quantitative properties of accretion shocks. We demonstrated the impact of environmental effects by exploring three models for the cosmic shock population. The first used the PressSchechter mass function to describe the underlying population of collapsed, accreting objects. All such objects were assumed to accrete material of the same density and temperature. This was our "control" model, which did not include any environmental effects. The second model used the double distribution of collapsed structures (Pavlidou \& Fields 2005) to describe the distribution of accreting objects with respect to both their mass and local environment overdensity or underdensity. The overall mass distribution of objects is the same as in the first model, as the double distribution integrates to the Press-Schechter mass function. This model then only included environmental effects caused by fluctuations in the primordial density field. Our third model was a refinement of model 2 , which considered all objects predicted by the double distribution to reside inside overdensities to accrete gas preheated and compressed in cosmic filaments.

The inclusion of primordial density fluctuations resulted to broader distributions with respect to Mach number of the mass current and kinetic power processed by accretion shocks, and an overall increase in the amplitude of such distributions, owing to an increase in the density of accreted material by the most massive objects, which dominate especially the processed kinetic power. Perhaps the most striking effect of accounting for this environmental factor is the separation of a second, high Mach number peak in the kinetic power distribution at low redshifts, due to an increasing number of higher-mass structures concentrating inside underdensities.

The inclusion of filament preheating and compression further increased the amplitude of the mass current and kinetic power distributions, and shifted the distributions towards lower Mach numbers. A more refined filament model, the need of which is underlined by our results, is expected to enhance these trends even more. Our treatment of both environmental factors has been systemati- 
cally conservative in estimating the impact of the effects on the shock properties. Consequently, we expect that these differences will be evident at least qualitatively in full numerical models.

The integrated kinetic power processed by shocks peaks at a redshift of $\sim 1$, while the integrated mass current peaks at higher redshifts, $z \sim 2$ (for model 3 ). The effect of the local environment is to increase the overall level of the processed energy at peak redshifts by a factor of $\sim 10$ for each environmental factor included.

Comparing the energy processed by cosmic accretion shocks to other energy inputs to the intergalactic medium, we find that the energy input of accretion shocks (as predicted by model 3) is higher that that of Type II supernovae for all $z \lesssim 3$, and it becomes more than an order of magnitude higher in the local universe. In addition, we found that energy processed by accretion shocks alone becomes comparable to the energy needed to reionize the universe by $z \sim 3.5$.

Numerical and observational tests of our model will quantify the importance of accretion shocks and their prominence among cosmic shock mechanisms. Our emphasis on accretion due to diffuse matter is complementary to minor mergers, thus comparison with simulations will shed light on the relative importance of these two components in the $\Lambda \mathrm{CDM}$ scenario. To compare the present results with simulation is however neither trivial nor immediate, because simulations include merger and filament shocks as well, but moreover because accretion shocks as we have defined them do not uniquely map onto the classification schemes used in the existing literature. A detailed, self-consistent comparison with simulations will appear in future work.

Of course, the question of the nature of cosmic shocks and the importance of accretion processes ultimately will be resolved observationally. Galaxy clusters provide a promising site to study accretion shocks (e.g. Lieu et al. 1996; Ensslin et al. 1998; Fusco-Femiano et al. 1999; Fujita \& Sarazin 2001; Govoni et al. 2001; Miniati et al. 2001a,b; Bagchi et al. 2002; Berrington \& Dermer 2003; Feretti et al. 2004; Gabici \& Blasi 2004; Keshet et al. 2004; Kocsis et al. 2005) As with simulations, there is a need to separate merger versus accretion components; this might be done spatially (e.g. Inoue et al. 2005), with accretion shocks dominating peripheral emission, particularly that due to inverse Compton. In addition, detection of $\gamma$-ray emission unambiguously associated with structure formation shocks, either by $\mathrm{TeV}$ (such as CANGAROO, HESS, MAGIC and VERITAS) or GeV (such as AGILE and GLAST) gamma-ray telescopes, will provide invaluable insight not only in the nature of the accretion process itself, but also the currently elusive subjects of the acceleration efficiency associated with cosmic shocks, as well as the properties of large-scale magnetic fields.

We thank Tom Abel, Susumu Inoue, Tom Jones, Hyesung Kang, Francesco Miniati, Temelachos Mouschovias, Tijana Prodanović, Kostas Tassis, Ben Wandelt, Ellen Zweibel, and an anonymous referee for useful discussions. This work was supported by the National Science Foundation through grant AST-0092939. The work of V.P. was partially supported by Zonta International through an Amelia Earhart Fellowship. V.P. additionally acknowledges support by the Kavli Institute for Cosmological Physics through grant NSF PHY-0114422.

\section{REFERENCES}

Bagchi, J., Enßlin, T. A., Miniati, F., Stalin, C. S., Singh, M., Raychaudhury, S., \& Humeshkar, N. B. 2002, New Astronomy, 7,249

Berrington, R. C. \& Dermer, C. D. 2003, ApJ, 594, 709

Bertschinger, E. 1985a, ApJS, 58, 39

Bertschinger, E. 1985b, ApJS, 58, 1

Brunetti, G., Setti, G., Feretti, L. \& Giovannini, G. 2001, NewA, 6,1

Brunetti, G., Blasi, P., Cassano, R. \& Gabici,S. 2004, MNRAS, 350,1174

Cen, R. \& Ostriker, J. P. 1999, ApJ, 514, 1

Cyburt, R. H., Fields,B. D. \& Olive, K. A. 2003, PhLB 567, 227

Davé R. et al. 2001, ApJ, 552, 473

Ensslin, T. A., Biermann, P. L., Klein, U., \& Kohle, S. 1998, A\&A, 332,395

Fang, T., Bryan, G. L., Bryan, \& Canizares, C.R. 2002, ApJ, 564, 604

Feretti, L., Burigana, C., \& Enßlin, T. A. 2004, New Astronomy Review, 48, 1137

Finoguenov, A., Briel, U. G. \& Henry, J. P. 2003, A\&A, 410, 777

Fujita, Y., \& Sarazin, C. L. 2001, ApJ, 563, 660

Fukugita, M., Hogan, C. J. \& Peebles, P. J. E. 1998, ApJ, 503, 518

Furlanetto, S. R. \& Loeb, A. 2004,ApJ611, 642

Gabici, S. \& Blasi, P. 2003a, ApJ, 583, 695

Gabici, S. \& Blasi,P. 2003b, APh, 19, 679

Gabici, S. \& Blasi, P. 2004, APh, 20, 579

Govoni, F., Feretti, L., Giovannini, G., Böhringer, H., Reiprich, T. H., \& Murgia, M. 2001, A\&A, 376, 803

Hegyi, D. J. \& Olive, K. A. 1986, ApJ, 303, 56

Hellsten, U., Gnedin, N. Y. \& Miralda-Escudé, J. 1998, ApJ509, 56

Inoue, S. \& Nagashima, M. 2005, AIP Conf. Proc. 745: High Energy Gamma-Ray Astronomy, 745, 567

Inoue, S., Aharonian, F. A., \& Sugiyama, N. 2005, ApJ, 628, L9
Kang, H. \& Jones, T. W. 2005, ApJ, 620, 44

Kang, H., Ryu, D., Cen, R. \& Song, D. 2005, ApJ620, 21

Keshet, U., Waxman, E., Loeb, A., Springel,V. \& Hernquist, L. 2003, ApJ, 585, 128

Keshet, U., Waxman, E., \& Loeb, A. 2004, New Astronomy Review, 48,1119

Kocsis, B., Haiman, Z., \& Frei, Z. 2005, ApJ, 623, 632

Kuo, P. H., Bowyer, S. \& Hwang, C.-Y. 2005, ApJ, 618, 675

Lacey, C. \& Cole, S. 1993, MNRAS, 262, 627

Lieu, R., Mittaz, J. P. D., Bowyer, S., Lockman, F. J., Hwang, C.-Y., \& Schmitt, J. H. M. M. 1996, ApJ, 458, L5

Loeb, A. \& Waxman, E. 2000, Nature, 405, 156

Mathur, S., Weinberg, D. H. \& Chen, X.2003, ApJ, 582, 82

Miniati, F., Ryu, D., Kang, H., Jones, T. W., Cen, R. \& Ostriker, J. P. 2000, ApJ, 542, 608

Miniati, F., Jones, T. W., Kang, H. \& Ryu,D. 2001a, ApJ562, 233

Miniati, F., Ryu, D., Kang, H. \& Jones, T. W. 2001b, ApJ, 559, 59

Miniati, F. 2002, MNRAS, 337, 199

Miniati, F. 2003, MNRAS, 342, 1009

Miniati, F., Ferrara, A., White, S. D. M., \& Bianchi, S. 2004, MNRAS, 348, 964

Nath, B. B., \& Silk, J. 2001, MNRAS, 327, L5

Nicastro F. et al. 2002, ApJ, 573, 157

Nicastro F. et al. 2005, Nature, 433, 495

Pavlidou, V. \& Fields, B.D. 2005, Phys. Rev. D, 71, 043510

Perna R. \& Loeb, A. 1998, ApJ, 503, L135

Press, W.H. \& Schechter, P. 1974, ApJ, 187, 425

Prodanović, T. \& Fields, B. D. 2004, ApJ, 616, L115

Prodanović, T. \& Fields, B. D. 2005, ApJ, 623, 877

Reimer, O., Pohl, M., Sreekumar P. \& Mattox, J. R. 2003, ApJ, 588 ,

Ryu, D. \& Kang, H. 1997, MNRAS, 284, 416 
Ryu, D., Kang, H., Hallman E. \& Jones, T. W. 2003, ApJ, 593, 599

Scharf, C. A. \& Mukherjee,R. 2002, ApJ, 580, 154

Spergel, D. N. et al. 2003, ApJS, 148, 75

Suzuki, T. K. \& Inoue, S. 2002, ApJ, 573, 168
Suzuki, T. K. \& Inoue, S. 2004, PASA, 21, 148

Totani, T. \& Kitayama, T. 2000, ApJ, 545, 572

Totani, T. \& Inoue, S. 2002, APh, 17, 79 